\title{
Inductions of recoveries from injuries to the cortex: Dissociation of equipotential and regionally specific mechanisms
}

\author{
MARK D. CLOUD, DONALD R. MEYER, and PATRICIA M. MEYER \\ Ohio State University, Columbus, Ohio
}

\begin{abstract}
Rats were first trained on a black-white discrimination problem and were then subjected to either one-quadrant or two-quadrant neocortical injuries. Following a test of retention, the subjects again sustained one-quadrant or two-quadrant lesions, always such that one quadrant remained intact. Another test of retention was administered, and then some subjects were completely decorticated by removal of the final remaining quadrant, followed by a final retention test. The effects on retention following the first-stage lesions suggested the existence of an equipotential, cortically related retrieval mechanism. The deficits in performance following secondstage injuries suggested the existence of a second, independent, posteriorly specific mechanism. The findings demonstrated that not only does interoperative retraining facilitate retrieval of preoperatively established memories, but also that injuries to any component of the cortex will induce reorganizations that will then prevent memory-retrieval deficits after any combination of subsequent injuries to the cortex.
\end{abstract}

Lashley (1935), in a classical investigation, examined the effects of neocortical ablations on learning and relearning of a black-white discrimination problem. He observed that performance on the problem is cortically dependent if the cortex is intact. $\mathrm{He}$ also observed that rats with injuries to the visual or posterior neocortex are able to learn the task as quickly as normal animals, and that lesions of the extravisual or anterior cortex have no effect on postoperative performance of the task. Hence, he concluded that training on the problem results in the storage of a memory by the cortex, provided that the cortex is intact, but if the cortex is not intact, the trace or engram is formed by subcortical systems.

Lashley's basic paradigm has since been utilized by many other students of recoveries of functions after injuries to the cerebral cortex. Several have confirmed his observation that ablations of the posterior neocortex will completely suppress postoperative performance on the black-white problem (e.g., Glendenning, 1972; Gray \& Meyer, 1981; Horel, Bettinger, Royce, \& Meyer, 1966; LeVere \& Morlock, 1973; Meyer, Yutzey, Dalby, \& Meyer, 1968; Petrinovich \& Carew, 1969; Thompson, 1960), but that such injuries have a very small effect upon the rate at which the problem is learned by preoperatively naive subjects (Bodart, Hata, Meyer, \& Meyer, 1980; Horel et al., 1966; Jonason, Lauber, Robbins, Meyer, \& Meyer, 1970; see Gray \& Meyer, 1981, for review). Indeed, the sole discrepancy between his ob-

This research was supported by a grant in aid from the Donald Jansen Fund of Ohio State University. servations and those of recent workers in the field is that it is now clear that anterior injuries will also affect postoperative performance on the problem (Hata, Diaz, Gibson, Jacobs, Meyer, \& Meyer, 1980; Horel et al., 1966; Kircher, Braun, Meyer, \& Meyer, 1970; Meyer et al., 1968; Meyer, Yutzey, \& Meyer, 1966).

It is equally clear that Lashley misinterpreted the nature of the deficits of posterior subjects, since current evidence suggests that a trained bilateral posterior preparation does not lose a memory for a problem that was formed prior to surgery. For example, if such a subject is retrained while being treated with amphetamine, the drug will facilitate retrieval of a black-white problem. Conversely, the same treatment administered to naive posterior preparations does not have any ameliorative effects on learning. Thus, injections of amphetamine appear to facilitate a rat's ability to regain access to an already existing memory (Braun, Meyer, \& Meyer, 1966). Moreover, even though subjects with posterior injuries require about as many trials to relearn the black-white problem as it takes them to learn it after surgery, the animals are very slow to learn a reversal of the problem that they have learned prior to surgery (LeVere \& Davis, 1977; LeVere \& Morlock, 1973; Meyer \& Meyer, 1982). These data also suggest that memory traces still exist and will strongly interfere with postoperative learning. And, finally, if trained posterior preparations are treated with 8-azaguanine, a drug that retards initial learning on the problem but not on remembering, the treatments permit the subjects to relearn the problem quickly (Davis \& LeVere, 1979; LeVere \& Fontaine, 1978). Therefore, the evi- 
dence at hand suggests that the memories are stored by subisocortical systems, regardless of whether or not the cortex has been injured.

Bilateral ablations of the posterior neocortex-that is, of the zones that have been shown to receive visual projections from the lateral geniculate nuclei and/or the lateral posterior thalamic nuclei (Hughes, 1977)bring about impairments of performance on the black-white problem that have proved to be precisely measurable. Thus, if the subjects are trained and retrained with the massed-trial procedures of Horel et al. (1966), the typical subject will relearn the problem in approximately 50 trials (see Barbas \& Spear, 1976). If, instead, the subjects are trained and retrained with the spaced-trial procedures of Glendenning (1972), the typical subject will relearn the problem in approximately 25 trials. Those values have been determined through studies of hundreds of posterior preparations (see Gray \& Meyer, 1981, for review), and in most of those studies the means for the values have proved to have a standard error of about $10 \%$.

Importantly, bilateral posterior ablations have the same effects upon postoperative performance regardless of whether the injuries are inflicted in one stage or two successive stages (Kircher et al., 1970; Petrinovich \& Carew, 1969). Hence, unless other treatments are employed, there is no "serial lesion effect" (Finger, 1978; Finger, Walbran, \& Stein, 1973). However, if two-stage posterior preparations are given interoperative retraining, they then exhibit sparing of ultimate performance on the black-white problem (Glendenning, 1972; Gray \& Meyer, 1981; Thompson, 1960). That effect is not due simply to extra training, since equivalent amounts of overtraining prior to surgery fail to yield it (Glendenning, 1972; Meyer \& Meyer, 1977). Also, the sparing is not observed unless the subjects are trained on the probmen before their first surgeries (Bodart et al., 1980). Consequently, the observed sparing is a facilitation of recall rather than a consequence of learning a new memory. Finally, the effect is explicitly a function of retraining on the black-white problem, since ultimate retention is not observed if the subjects are trained on other kinds of visual tasks between the operations (Davis \& LeVere, 1982; Gray \& Meyer, 1981).

Now we shall consider the performances of subjects with one-stage bilateral ablations of the extravisual cortex. If the animals are retrained with the massed-trial procedure, they relearn in 35 trials (Horel et al., 1966); if retrained with the spaced-trial procedure, they relearn in 17 trials (Hata et al., 1980). Hence, despite the fact that the subjects are quicker to relearn than posterior preparations, the relative values of the two varieties of relearning scores are the same. That is, the scores of anterior subjects, regardless of the method one employs, are about two- thirds of the scores for subjects with bilateral posterior injuries.

However, we also have a large amount of data that indicate that unilateral first-stage injuries to either the visual or extravisual cortex are followed by impairments that can be corrected by eight or nine spaced retraining trials (Gray \& Meyer, 1981; Meyer \& Meyer, 1977). The standard errors of the scores for such subjects are approximately \pm 1 trial, and the means are insensitive to small variations in the scopes of the injuries to the brain. The deficits are not attributable to surgical trauma, since sham preparations will relearn the problem in approximately one or two trials (Dewberry, Note 1). Hence, although bilateral posterior preparations have greater impairments than bilateral anterior preparations, an injury to a single quadrant of the cortex has the same effect upon performance of the problem regardless of the locus of the injury.

The consequence of a first-stage injury to a quadrant of the cortex is interesting because the eight or nine trials that the subjects require are half of the 17 trials that are required for relearning the problem by bilateral anterior preparations. Such results have prompted Meyer and Meyer (1982) to propose that, despite the well-established regional specializations of various subsectors of the cortex, a function exists for which the cortex is equipotential. Also, they have argued that the posterior neocortex has a regionally specific function that cannot be detected through assessments of performance on the black-white problem unless and until the entire posterior cortex is destroyed. According to the theory, the deficit induced by completion of a posterior injury is about eight trials, and that impairment, when added to the 17-trial impairment of subjects with injuries to any two quadrants of the cortex, accounts for the fact that a one-stage bilateral posterior preparation will relearn the problem in about 25 spaced trials.

The argument is also based upon the fact that a second-stage serial posterior ablation that completes the destruction of the visual neocortex induces an impairment that can be corrected by eight trials of training, provided that the subjects had also been given interoperative retraining on the problem (Bodart et al., 1980; Meyer \& Meyer, 1977). In contrast, a second-stage anterior injury that completes the destruction of the extravisual cortex has almost no additional effect (Glendenning, 1972). Hence, it appears that interoperative retraining permits compensations for impairments of retrieval that are brought about by injuries to the equipotential mechanism but not for the regionally specific impairments that are brought about by injuries that destroy or complete the destruction of the posterior cortex.

It has also been shown that if naive rats with firststage bilateral anterior ablations that are trained on 
the problem and are then subjected to second-stage bilateral posterior ablations, they relearn the problem as quickly as subjects that are trained as normals and are then prepared with first-stage bilateral posterior ablations (Hata et al., 1980; Horel et al., 1966). Moreover, if naive posterior preparations are trained on the problem and are then subjected to anterior ablations that complete the destruction of the entire neocortex, the animals will relearn the problem as quickly as preoperatively trained bilateral anterior preparations (Horel et al., 1966; Howarth, Meyer, \& Meyer, 1979). Hence, in both instances, the twostage subjects are not disadvantaged by their having lost the entire neocortex, but neither does the training they receive between the surgeries protect performance on the problem following the second-stage ablations.

Importantly, however, the two-stage subjects were not trained before their first surgeries. According to the theory of Meyer and Meyer (1982), injuries to the equipotential mechanism for retrieval cannot be expected to induce compensations for impairments of its function if the subject has no memory to remember. Also, the theory suggests that any injury to the equipotential mechanism should permit the compensations to occur regardless of the scopes and the sites of the injury. The present study was conducted to provide a rigorous test of those ideas.

The thrust of the study was as follows. First, we reexamined the accuracy with which the law of mass action predicts the effects of first-stage injuries to one or two quadrants of the cortex, provided, once again, that the two-quadrant injuries do not destroy the entire posterior cortex. Second, the animals were subjected to ablations in which the remainder of the cortex was destroyed except for a quadrant of the anterior or the posterior cortex. Then, after measures of the second-stage effects had been obtained, a number of the animals were subjected to ablations that completed the destruction of the entire neocortex and were once again tested for performance.

The quantitative predictions from the theory of Meyer and Meyer (1982) were as follows. The subjects with first-stage one-quadrant injuries, regardless of whether the injuries were visual or extravisual, were expected to relearn in eight or nine mean spaced trials. The subjects with first-stage two-quadrant injuries, in any combination that does not bilaterally destroy the posterior quadrants, were expected to relearn the problem in 17 mean spaced trials. The second-stage effects were expected to be completely independent of the nature of the first-stage injuries, but to vary as a function of whether or not those injuries bilaterally destroyed or completed the bilateral destruction of the visual neocortex. In the latter circumstance, the subjects were expected to relearn the problem in approximately eight spaced trials, regardless of whether the second-stage ablation was a one- or two-quadrant injury. The grounds for that prediction were that interoperative retaining on the task would induce a compensation such that further injuries to the equipotential retrieval mechanism would be without effect upon retention. Subjects with secondstage injuries that spared a quadrant of the posterior cortex, and hence would not suffer the eight-trial regionally specific deficit, were expected to relearn the task very quickly. We also predicted that animals prepared with third-stage ablations of the cortex would re-relearn the problem very quickly if the injury destroyed a still-intact anterior quadrant, but that animals prepared with third-stage ablations that destroyed a remaining posterior quadrant would require about eight further trials to re-relearn the problem.

\section{METHOD}

\section{Subjects}

Male Long-Evans hooded rats, $90-120$ days of age at the beginning of training, were employed. The 44 subjects were housed in individual cages and were maintained on an ad-lib food and water schedule.

\section{Apparatus and Training Procedures}

Training was conducted in a version of the Thompson-Bryant (1955) apparatus, which consisted of a startbox, a choice compartment, and a goalbox. A vertical partition with two square openings separated the choice compartment from the goalbox. These openings were fitted with black and white stimulus doors with approximate luminances of .1 and $3.0 \mathrm{~mL}$, respectively. The walls of the apparatus as well as the vertical partition were constructed of black Plexiglas. Small fluorescent lamps, mounted on transparent lids, illuminated the start and choice compartments. The floors of the start and choice compartments were made of stainless steel bars from which weak motivating shocks could be delivered.

The animals were first handled for at least $5 \mathrm{~min}$ per day for 5 days. On Day 6, each animal was permitted to explore all areas of the apparatus for $15 \mathrm{~min}$. Next, on Day 7, pretraining began, and each subject was trained to run into the goalbox to avoid mild foot shocks. Pretraining doors with black and white oblique stripes were generally fitted into the square openings of the partition so that the rat knocked down one of the pretraining doors to enter the goalbox. On Day 8, preoperative training was begun, and the doorways were fitted with the black and white stimulus doors. The spaced-trial training method of Glendenning (1972) was used, which limited the number of trials to 25 per day with a 10 -min rest following the 8 th and 16 th trials. The correct stimulus throughout the study was the white stimulus whose left-right position was governed by a Gellerman (1933) series. A latch at the rear locked the incorrect black door, requiring the subject to enter the goalbox only by knocking down the correct white stimulus. Training continued until the subject had reached a criterion of 9 correct responses in 10 trials.

\section{Experimental Design}

All of the subjects received preoperative training on the blackwhite problem. Following each stage of the serial ablations and a 12-day recovery interval, the animals were all retrained on the task to the preoperative criterion of performance. Each group was equally divided with respect to the side of the brain sustaining the injury. In Phase 1, we measured impairments of performance following one-quadrant and two-quadrant injuries of the cortex. In Phase 2, we measured the effects of interoperative retraining upon performance of a second test following second-stage ablation 
of one or two additional quadrants of the cortex. Phase 3 was designed to assess the effects of a third serial ablation of the stillremaining quadrant of the cortex.

Descriptions of the various groups of subjects that were studied utilize the following abbreviations: Unilateral ablations of one quadrant are signified $\mathrm{v}$ (visual) and a (anterior), while $\mathrm{V}$ denotes simultaneous bilateral visual decortication. When lesions involve both anterior and posterior sectors, $\mathrm{C}$ and I signify whether the quadrants are contralateral or ipsilateral to each other. Hence, a rat subjected to an aVa treatment is first given training on the black-white discrimination problem (L). Next, the animal sustains a unilateral anterior ablation (a). A retention test $\left(R_{1}\right)$ is then conducted, followed by a simultaneous bilateral visual ablation (V). The animal is trained once again $\left(\mathbf{R}_{\mathbf{2}}\right)$ and then subjected to a unilateral anterior injury (a). Finally, a third retention test $\left(\mathbf{R}_{\mathbf{3}}\right)$ is conducted. Table 1 outlines the behavioral and surgical manipulations of each of the five groups.

\section{Surgical Procedures}

The surgeries were performed with clean technique while the rats were under deep anesthesia induced by sodium pentobarbital. The ablations were accomplished with the pia-stripping method in conjunction with aspiration at the margins of the injuries (see Meyer \& Meyer, 1971). Bregma was taken as the landmark for the medial point of demarcation between an anterior and posterior ablation. Thus, one-quadrant anterior ablations were intended to extend from the bregmoid suture to the tip of the frontal pole and from the sagittal sinus to the rhinal fissure. The posterior lesions were intended to extend from the bregmoid suture to the occipital pole and from the sagittal sinus to the rhinal fissure. Following the surgeries, the subjects were given a broadband antibiotic.

\section{Histological Procedures}

Because of the animals' unique status of chronic complete decortication, 15 subjects were saved for future experimentation. The remaining 29 subjects following the last retention test were given lethal doses of sodium pentobarbital and were then perfused through the heart with normal saline followed by $10 \%$ Formalin. The extents of the cortical lesions on the surface were drawn on standard Lashley diagrams. The brains were frozen and sectioned at $40 \mu$. Every 10th section through the cortical lesion and every 5 th section through the dorsal lateral geniculate nucleus were mounted and stained with cresyl violet. Thereafter, the dorsal lateral geniculate nuclei were studied to determine the extent of retrograde degeneration.

\section{RESULTS}

\section{Histological Results}

The extent of neocortical damage was comparable in size to that of Meyer, Johnson, and Vaughn (1970) and Beattie, Gray, Rosenfield, Meyer, and Meyer (1978). ${ }^{1}$ Examination of the dorsal thalamus in those animals with complete removal of the neocortex revealed massive degeneration throughout the dorsal thalamus. When sparing of normal cells did occur, it was most often noted in the dorsolateral portion of the dorsal lateral geniculate nuclei, medial geniculate bodies, portions of the anterior thalamic nuclei, and nucleus posterior lateralis. We also commonly observed concentrated regions of necrotic tissue, which Braun (1975) attributes to incidental vascular damage incurred during the decortication procedure. Typically, it extends throughout the rostralcaudal extent of the thalamus and in the same relative position into the caudate nucleus.

In the group with an intact posterior quadrant of the neocortex remaining, there was no thalamic cell degeneration on the side of the remaining cortex in dorsal lateral geniculate and nucleus posterior lateralis. The extent of spared cortical tissue on these animals compared well in scope with the size of onequadrant ablations in other studies (Glendenning, 1972; Ritchie, 1973).

\section{Behavioral Results}

Phase 1. Table 2 presents the means, the standard errors of the means, and the medians for the $L$ and $R_{1}$ scores of all the groups. The overall mean for $L$ was 19.0. There were no significant differences between $L$ scores for any of the treatment groups as assessed by a one-way analysis of variance $[F(4,39)$ $=.22]$.

Table 1

Behavioral and Surgical Manipulations for All Groups

Group $\quad$ TRAIN


Table 2

Learning and Relearning $\left(R_{1}\right)$ Scores for All Groups

\begin{tabular}{|c|c|c|c|c|c|c|}
\hline GROUP & $\mathrm{N}$ & Mean & $\pm \quad$ LE/Mdn & $\mathrm{N}$ & Mean & $\pm{ }^{\mathrm{R}}{ }_{\text {SE/Mdn }}$ \\
\hline Op. 1: One-Quadrant Injuries & 13 & 18.5 & $\pm 2.0 / 18.0$ & 13 & 8.1 & $\pm \quad 1.4 / 10.0$ \\
\hline $\mathrm{aVa}$ & 7 & 17.4 & $\pm 2.6 / 18.0$ & 7 & 5.9 & $1.5 / 4.0$ \\
\hline $\mathrm{vCa}$ & 6 & 19.8 & $\pm 3.3 / 17.0$ & 6 & 10.7 & $2.1 / 12.0$ \\
\hline Op. 1: Two-Quadrant Injuries & 31 & 19.2 & $\pm 1.6 / 17.0$ & 31 & 15.5 & $1.1 / 15.0$ \\
\hline $\operatorname{Iax}$ & 12 & 17.8 & $\pm 1.7 / 16.0$ & 12 & 13.2 & $1.6 / 12.5$ \\
\hline Iva & 6 & 19.7 & $\pm 4.9 / 13.5$ & 6 & 13.0 & $1.2 / 14.0$ \\
\hline $\mathrm{Cav}$ & 13 & 20.3 & $\pm 2.7 / 18.0$ & 13 & 18.8 & $1.8 / 19.0$ \\
\hline
\end{tabular}

Note-Highlighted areas refer to scores for combined groups in Phase 1.

The two groups that sustained first-stage onequadrant injuries required a mean of 8.1 trials to relearn the task. Unilateral posterior ablations were performed on Group vCa $(\mathrm{N}=6)$. These animals relearned the problem in 10.7 trials. Group aVa $(\mathrm{N}=7)$ sustained unilateral anterior lesions and reached the $9 / 10$ criterion in 5.9 trials. This retention score was not significantly different from Group vCa $(t=1.90$, $\mathrm{p}<.05)$.

Group Cax $(\mathrm{N}=13)$, which was subjected to crossed ablations of the anterior and posterior quadrants, relearned the problem in $\mathbf{1 8 . 8}$ mean trials. Two groups, Iax and Iva sustained ipsilateral anterior-posterior injuries $(\mathrm{N}=18)$ and rereached criterion in 13.1 spaced. trials. The difference in $\mathbf{R}_{\mathbf{1}}$ scores between these two types of two-quadrant injuries was statistically significant $(t=2.9, p<.01)$. The overall mean for the 31 subjects with two-quadrant first-stage injuries was 15.5 trials.

Phase 2. Table 3 presents $R_{1}$ and $R_{2}$ scores. It combines groups that sustained second-stage injuries to an anterior quadrant and groups that were subjected to second-stage injuries that completed the destruction of the posterior neocortex. The $\mathbf{R}_{\mathbf{2}}$ scores for the two combined groups were 3.6 and 8.9 spaced trials, respectively. The difference was statistically significant $(t=5.3, p<.001)$. The intragroup differences were not significant within the anterior group

Table 3

Relearning $\left(\mathbf{R}_{1}\right)$ and Relearning $\left(\mathbf{R}_{2}\right)$ Scores for All Groups

\begin{tabular}{|c|c|c|c|c|c|c|}
\hline GROUP & $\mathrm{N}$ & Mean & $\pm{ }^{R_{1}}{ }_{S E / M d n}$ & N & Mean & $\pm{ }^{\mathrm{R}_{2}} \mathrm{SE} / \mathrm{Maln}$ \\
\hline Op. 2: Completing Posterior & & & & 18 & 8.9 & $.68 / 8.0$ \\
\hline Iva & 6 & 13.0 & $\pm 1.2 / 14.0$ & 6 & 10.2 & $1.6 / 9.5$ \\
\hline $\mathrm{aVa}$ & 7 & 5.9 & $\pm 1.5 / 4.0$ & 6 & 7.3 & $.76 / 7.0$ \\
\hline$v \mathrm{Ca}$ & 6 & 10.7 & $\pm 2.1 / 12.0$ & 6 & 0.2 & $.91 / 9.0$ \\
\hline 0p. 2: Posterior Present & & & & 24 & 3.6 & $.70 / 3.0$ \\
\hline $\operatorname{Iax}$ & 12 & 13.2 & $+1.6 / 12.5$ & 12 & 2.8 & $.87 / 2.5$ \\
\hline Cav & 13 & 18.8 & $\pm 1.8 / 19.0$ & 12 & 4.4 & $1.1 / 4.0$ \\
\hline
\end{tabular}

Note-Highlighted areas refer to scores for combined groups in Phase 2. 
Table 4

Relearning $\left(R_{2}\right)$ and Relearning $\left(R_{3}\right)$ Scores for All Groups

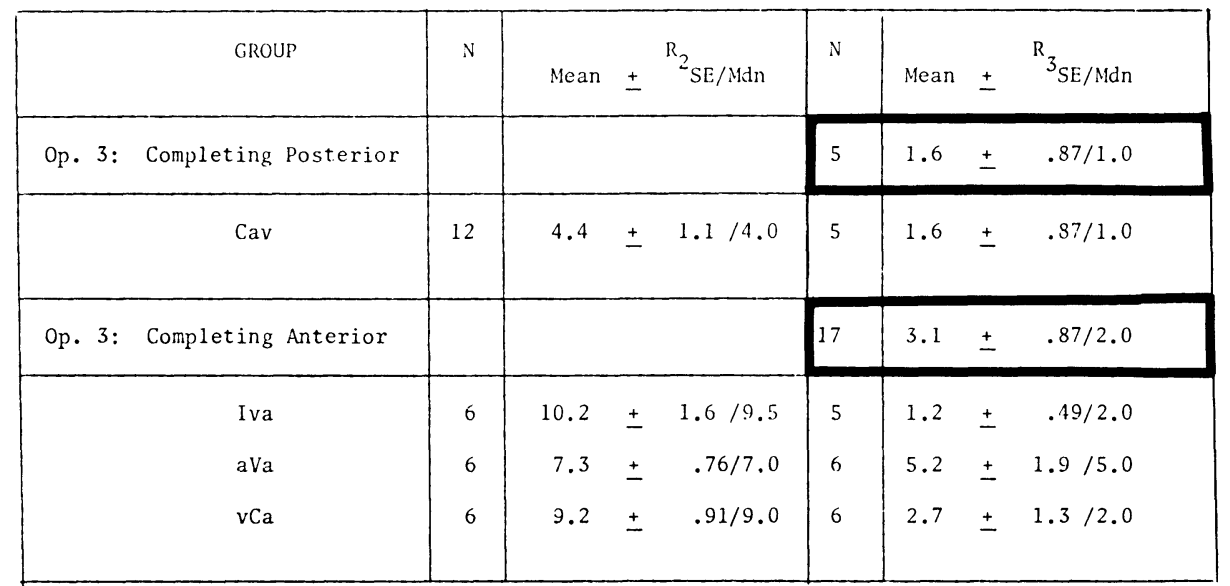

Note-Highlighted areas refer to scores for combined groups in Phase 3.

$(\mathrm{t}=1.14, \mathrm{p}<.05)$ or within the completing posterior group $[F(2,15)=1.59]$.

Phase 3. Table 4 presents $R_{2}$ and $R_{3}$ scores for the four groups of subjects that sustained third-stage injuries that completed the destruction of the neocortex. Group Cav, which was subjected to a third stage completing visual cortical ablation $(\mathrm{N}=5)$, re-relearned the problem in 1.6 mean spaced trials. Three groups that sustained ablations that destroyed the remaining anterior quadrant re-relearned the problem in 3.6 mean spaced trials. The differences among any of the groups were not statistically significant $[F(3,18)=$ $1.81]$.

\section{DISCUSSION}

First, we shall discuss the fates of our predictions from the theory of Meyer and Meyer (1982). The theory predicted that the relearning scores for subjects with ablations of one quadrant of the cortex would have a mean of eight to nine spaced trials. The observed mean for the 13 one-quadrant subjects that were retrained during Phase 1 was 8.1 spaced trials. The finding is important because it replicates and confirms the deficit previously found following onequadrant cortical ablations (Gray \& Meyer, 1981; Meyer \& Meyer, 1977), and it shows once again that the scores we employ in our quantitative reasoning are reliable.

The prediction for first-stage two-quadrant subjects was that those animals would relearn the task in about 17 spaced trials. However, the theory also predicted that animals with two-quadrant crossed ablations would relearn the problem at about the same rate as subjects with ipsilateral injuries. The theory overestimated the effects of ipsilateral injuries by four spaced trials and underestimated the effects of crossed-quadrant injuries by 1.8 trials. The intergroup difference was significant and possibly reflects an effect of unilateral vs. bilateral severances of intrahemispheric pathways between the posterior and the anterior cortex. The question is deserving of additional study, but the findings, as a whole, demonstrate that the law of mass action is a very good predictor of the consequences of first-stage ablations that spare at least one posterior quadrant.

We next discuss the outcomes of the Phase 2 procedures. All of the aimals had by then been prepared with serial ablations that destroyed three quadrants of the cortex. The $R_{1}$ interoperative retraining was expected to correct the first-stage impairments and also to induce protection of performance following further injuries to the equipotential mechanism. Thus, subjects that still retained a posterior quadrant were expected to have negligible impairment following further damage. However, subjects with injuries that bilaterally destroyed or completed the destruction of the visual neocortex were expected to require approximately eight trials to recover from their regionally specific deficits. The 24 subjects with completing posterior injuries re-relearned in 8.9 trials, and the 18 subjects that still had a posterior quadrant re-relearned in 3.6 trials. We consider these outcomes to be strongly supportive of the theory.

We next discuss the Phase 3 results. At that stage, all of the Phase 3 subjects had undergone complete decortication. The predictions from the theory were that third-stage ablations of an anterior quadrant would have negligible effects on performance, while third-stage ablations of a posterior quadrant would still yield the regionally specific effect and the subjects would require eight trials. The prediction for the subjects with anterior lesions was fulfilled, but the posterior subjects also relearned the problem 
quickly. At present, we have no explanation for the latter observation and hence no defensible conception of the nature of the regionally specific impairment.

It is nonetheless apparent that the regionally specific impairment, regardless of its basis, is dissociable from impairments of the equipotential function. Also, injuries to the equipotential mechanism have first-stage effects that conform to the law of mass action. However, the observed impairment is not a loss of memory. Rather, it is an impairment of access to memories that are stored by subcortical systems, since injuries to the equipotential mechanism have effects that are completely determined by whether the subject has a memory to remember. We contrast our conclusions with Pribram's (1971) proposition that the cortex is a part of a distributed system that stores memory traces, and we will here reiterate our past conclusions that the cortex does not appear to be a memory bank (Meyer, 1972; Meyer \& Beattie, 1977; Meyer \& Meyer, 1982).

Our empirical supports for the conclusions we have drawn have properties in common with the arguments presented by Flourens (1842) and by Lashley (1929) for their theories of cerebral organization. Both Flourens and Lashley were impressed by the fact that, under at least some conditions, the behavioral effects of injuries to the cortex are functions of the extent, but not of the locus, of the injuries. We have also been impressed by the accuracy with which an animal's performance on the black-white problem following a first-stage injury to the cortex is predicted by the extent of the ablation and, in certain cases, is independent of the locus of the ablation. Although such predictions are off the mark for subjects with bilateral posterior injuries, they are accurate for even those preparations if we discount the regionally specific and dissociable effect of destruction of the posterior neocortex.

However, as Hunter (1931) first pointed out, the mere demonstration that a given impairment is a function of the scope of an injury to the cortex, but not of the locus of the injury, is not a compelling proof of the existence of an equipotential mechanism. Essentially, Hunter argued that performance of a task reflects a variety of functions and, therefore, that injuries of comparable extent to various subsectors of the cortex can have the same quantitative consequences, but for altogether different reasons. The findings of this investigation support his argument, for to it we can add that injuries of comparable extent and loci can also produce impairments that are qualitatively dissimilar and yet are quantitatively the same. Thus, it takes a serial posterior preparation about eight trials to recover from both its first-stage and second-stage ablations, but the numbers nonetheless are measures of disruptions of completely dissociable functions.
We will therefore stress our proof that the cortex is equipotential for a function does not rest solely on the kinds of arguments that were offered by Flourens (1842) and by Lashley (1929). It also has a third and, we believe, significant ingredient. Thus, we have also shown that first-stage injuries of various extents and placements will also permit interoperative retraining to induce compensations that are independent of the nature of the first-stage injury. In the past, we have thought that compensations for injuries to the visual neocortex are observed only if the first-stage ablations destroy at least a part of that subzone (Meyer \& Meyer, 1977), but the outcomes of this study have clearly demonstrated that anterior injuries will also initiate the process.

Perhaps the single most dramatic finding of this study was that unilateral anterior subjects that were tested after having been prepared with second-stage bilateral ablations of the posterior cortex (Group aVa) re-relearned the problem in a mean of 7.4 spaced trials. Hence the interoperative training they received induced a protection of ultimate retention of approximately 17 spaced trials, which, according to our theory, is the nonspecific cost of injuries to any two quadrants. As far as we can see, there is simply no way of accounting for a finding of that kind unless we assume that the anterior injury permitted compensations for impairments of a function that the cortex performs as a whole. Also, and notably, the compensations appear to be complete, regardless of the scope of the first-stage injury to the cortex, and hence, although the law of mass action is descriptive of first-stage effects, it does not apply to secondstage effects.

We believe that our conclusions bear on the question of why cerebral lesions that develop very slowly are often unproductive of compelling symptoms until the disease is well advanced (see Jackson, 1958). That phenomenon implies that damage to the brain permits compensations for the injuries and poses the question of what kinds of injuries permit what kinds of compensations. We suggest that a partial answer to the question is that any kind of injury to the neocortex will permit compensations for impairments of remembering which would otherwise be noted after any further injury to the cortex. We also suggest that the generalization is valid for many kinds of memories, for it seems to us unlikely that the brain would have an equipotential retrieval mechanism that would be concerned only with a rat's recall of a memory for the black-white problem.

\section{REFERENCE NOTE}

1. R. G. Dewberry, personal communication, February 1982.

\section{REFERENCES}

Barbas, H., \& Spear, P. D. Effects of serial unilateral and serial bilateral visual cortex lesions on brightness discrimination re- 
bilateral visual cortex lesions on brightness discrimination relearning in rats. Journal of Comparative and Physiological Psychology, 1976, 90, 279-292.

Beattie, M. S., Gray, T. S., Rosenfeld, J. A., Meyer, P. M., \& MEYER, D. R. Residual capacity for avoidance learning in decorticate rats: Enhancement of performance and demonstration of latent learning with d-amphetamine treatments. Physiological Psychology, 1978, 6, 279-287.

Bodart, D. J., Hata, M. G., Meyer, D. R., \& Meyer, P. M. The Thompson effect is a function of the presence or absence of preoperative memories. Physiological Psychology, 1980, 8, 15-19.

Braun, J. J. The neocortex and feeding behavior in the rat. Journal of Comparative and Physiological Psychology, 1975, 89, 507-522.

Braun, J. J., Meyer, P. M., \& Meyer, D. R. Sparing of a brightness habit in rats following visual decortication. Journal of Comparative and Physiological Psychology, 1966, 61, 79-82.

DAVIS, N., \& LEVERE, T. E. Recovery of function after brain damage: Different processes and facilitation of one. Physiological Psychology, 1979, 7, 233-240.

DAVIS, N., \& LEVERE, T. E. Recovery of function after brain damage: The question of individual behaviors or functionality. Experimental Neurology, 1982, 75, 68-78.

Finger, S. Lesion momentum and behavior. In S. Finger (Ed.), Recovery from brain damage. St. Louis, Mo: Plenum Press, 1978.

Finger, S., Walbran, B., \& Stein, D. G. Brain damage and behavioral recovery: Serial lesion phenomena. Brain Research, 1973, 63, 1-18.

Flourens, P. Recherches experimentales sur les propriétés et les fonctions du système nerveux. Paris: Author, 1842.

Gelle Rman, L. W. Chance orders of alternating stimuli in visual discrimination experiments. Journal of Genetic Psychology, 1933, 42, 207-208.

GLendenning, R. L. Effects of training between two unilateral lesions of visual cortex upon ultimate retention of black-white habits in rats. Journal of Comparative and Physiological Psychology, 1972, 80, 216-219.

GrAY, T. S., \& MEYER, D. R. Effects of mixed training and overtraining on recoveries from amnesias in rats with visual cortical ablations. Physiological Psychology, 1981, 9, 54-62.

Hata, M. G., Diaz, C. L., Gibson, C. F., Jacobs, C., Meyer, P. M., \& Meyer, D. R. Perinatal injuries to extravisual cortex enhance the significance of visual cortex for performance of a visual habit. Physiological Psychology, 1980, 8, 9-14.

Horel, J. A., Bettinger, L. A., Royce, G. J., \& Meyer, D. R. Role of neocortex in the learning and relearning of two visual habits by the rat. Journal of Comparative and Physiological Psychology, 1966, 61, 66-78.

Howarth, H., Meyer, D. R., \& Meyer, P. M. Perinatal injuries to the visual cortex enhance the significance of extravisual cortex for performances of a visual habit. Physiological Psychology, 1979, 7, 163-166.

Hughes, H. C. Anatomical and neurobehavioral investigations concerning the thalamo-cortical organization of the rat's visual system. Journal of Comparative Neurology, 1977, 175, 311-336.

Hunte R, W. S. A consideration of Lashley's theory of the equipotentiality of cerebral action. Journal of Genetic Psychology, $1930,3,455-468$.

JACKSON, J. H. Selected writings of John Hughlings Jackson. New York: Basic Books, 1958.

Jonason, K. R., Lauber, S., Robbins, M. J., Meyer, P. M., \& MEYER, D. R. The effects of dl-amphetamine upon discrimination behaviors in rats with cortical lesions. Journal of Comparative and Physiological Psychology, 1970, 73, 47-55.

Kircher, K. A., Braun, J. J., Meyer, D. R., \& Meyer, P. M. Equivalence of simultaneous and successive neocortical ablations in production of impairments of retention of black-white habits in rats. Journal of Comparative and Physiological Psychology, 1970, 71, 420-425.

LASHLEY, K. S. Brain mechanisms and intelligence. Chicago: University of Chicago Press, 1929.

LASHLEY, K. S. The mechanisms of vision: XII. Nervous structures concerned in habits based upon reactions to light. Comparative Psychology Monographs, 1935, 11, 43-79.

LEVERE, T. E., \& Davis, N. Recovery of function after brain damage: The motivational specificity of spared neural traces. Experimental Neurology, 1977, 57, 883-889.

LeVere, T. E., \& Fontaine, C. W. A demonstration of the importance of RNA metabolism for the acquisition but not performance of learned behaviors. Experimental Neurology, 1978, 59, 444-449.

LEVERE, T. E., \& MorLock, G. W. The nature of visual recovery following posterior decortication in the hooded rat. Journal of Comparative and Physiological Psychology, 1973, 83, 62-67.

Meyer, D. R. Access to engrams. American Psychologist, 1972, 27, 124-133.

Meyer, D. R., \& Be attie, M. S. Some properties of substrates of memory. In L. H. Miller, C. A. Sandman, \& A. J. Kastin (Eds.), Neuropeptide influences on brain and behavior. New York: Raven Press, 1977.

MEYER, D. R., \& MEyer, P. M. Dynamics and bases of recoveries of functions after injuries to the cerebral cortex. Physiological Psychology, 1977, 5, 133-165.

Meyer, P. M., Johnson, D., \& Vaughn, D. The consequences of septal and neocortical ablations upon learning a two-way avoidance response. Brain Research, 1970, 22, 113-120.

Meyer, P. M., \& MEYER, D. R. Neurosurgical procedures with special reference to aspiration lesions. In R. B. Myers (Ed.), Methods in neurobiology. New York: Academic Press, 1971.

MEYeR, P. M., \& MEYER, D. R. Memory, remembering and amnesia. In R. L. Isaacson \& N. E. Spear (Eds.), Expression of knowledge. New York: Plenum Press, 1982.

Meyer, D. R., Yutzey, D. A., \& Meyer, P. M. Effects of neocortical ablations on relearning of a black-white discrimination habit by two strains of rats. Journal of Comparative and Physiological Psychology, 1966, 61, 83-86.

Meyer, P. M., Yutzey, D. A., Dalby, D. A., \& Meyer, D. R. Effects of simultaneous septal-anterior and anterior-posterior lesions upon relearning a black-white discrimination. Brain Research, 1968, 8, 281-290.

Petrinovich, L., \& Carew, T. J. Interaction of neocortical lesion size and interoperative experience in retention of a learned brightness discrimination. Journal of Comparative and Physiological Psychology, 1969, 68, 451-454.

Pribram, K. H. Languages of the brain. Englewood Cliffs, N.J: Prentice-Hall, 1971.

RitchiE, G. D. A further investigation of the effects of occlusion on relearning of black-white habits by rats with simultaneous or successive neocortical ablations. Unpublished master's thesis, Ohio State University, 1973.

ThомpSON, R. Retention of a brightness discrimination following neocortical damage in the rat. Journal of Comparative and Physiological Psychology, 1960, 53, 211-215.

Thompson, R., \& BryanT, J. H. Memory as affected by activity of the relevant receptor. Psychological Reports, 1955, 1, 393-400.

\section{NOTE}

1. We apologize for not presenting the brain diagrams in a figure. The university temporarily put a freeze on all expenditures.

(Manuscript received March 3, 1982; accepted for publication March 3, 1982.) 